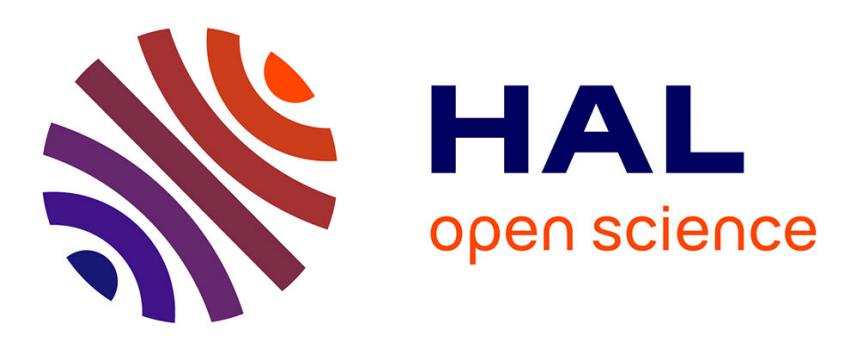

\title{
Bernadette Bensaude-Vincent, L'opinion publique et la science. À chacun son ignorance
}

\author{
Maël Dieudonné
}

\section{To cite this version:}

Maël Dieudonné. Bernadette Bensaude-Vincent, L'opinion publique et la science. À chacun son ignorance. 2013, http://lectures.revues.org/12348. halshs-00973385

\section{HAL Id: halshs-00973385 \\ https://shs.hal.science/halshs-00973385}

Submitted on 8 Apr 2014

HAL is a multi-disciplinary open access archive for the deposit and dissemination of scientific research documents, whether they are published or not. The documents may come from teaching and research institutions in France or abroad, or from public or private research centers.
L'archive ouverte pluridisciplinaire HAL, est destinée au dépôt et à la diffusion de documents scientifiques de niveau recherche, publiés ou non, émanant des établissements d'enseignement et de recherche français ou étrangers, des laboratoires publics ou privés. 


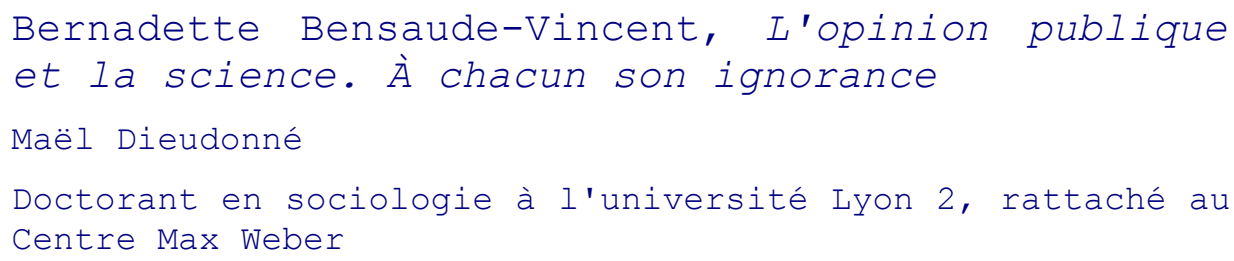

L'opinion mérite-t-elle le discrédit dont elle souffre aujourd'hui, à une époque où la seule connaissance légitime semble être scientifique ? Récusant la thèse de la rupture épistémologique, selon laquelle elles diffèrent ontologiquement, Bernadette Bensaude-Vincent propose dans cet ouvrage de réhabiliter l'opinion en révélant le processus historique ayant conduit à sa disqualification : c'est la science elle-même qui l'a construite comme un épouvantail, pour se renforcer en la dénonçant. Pour ce faire, elle adopte une démarche originale, consistant à ne plus analyser ces deux manières de connaître séparément et absolument, mais conjointement et historiquement. Les deux premiers chapitres sont consacrés à cette étude, tandis que le troisième examine quelques alternatives à la configuration actuelle des rapports entre la science et l'opinion, qui lui paraît dangereuse pour la démocratie.

Considérons d'abord son raisonnement historique. Son point de départ est le constat de l'ambivalence des rapports que la science entretient avec l'autorité : elle se présente à la fois comme une instance critique (elle défie les savoirs établis et les puissances sociales qui les portent) et comme une institution dogmatique (elle s'érige elle-même en force pour défendre l'orthodoxie de ses connaissances) ${ }^{1}$. Depuis la fin $\mathrm{du} \mathrm{XVIII}^{\mathrm{e}}$ siècle où l'auteur choisi de la faire commencer, l'histoire de la science face à l'opinion est marquée par cette ambiguïté. D'un côté, le public a joué un rôle fondamental dans l'avènement de la science moderne, en exigeant que les pratiques de recherches deviennent, précisément, publiques (à rebours de la tradition alchimique par exemple). Les échanges épistolaires notamment, grâce au procédé rhétorique élevant chaque correspondant au rang de témoin potentiel des expériences, contribuent à imposer l'idée que ces dernières doivent être reproductibles. Mais d'un autre côté, ce public rassemble seulement des membres de l'élite éclairée, qui s'efforcent de se distancier des croyances populaires (ainsi du mesmérisme, qu'un rapport de l'Académie des Sciences condamne fermement en 1784) ou de la science-spectacle (atteignant son paroxysme avec les expérimentations électriques de l'abbé Nollet). S'il s'élargit dans la seconde moitié du XIX $\mathrm{X}^{\mathrm{e}}$ siècle, c'est largement grâce à l'activisme commercial d'éditeurs soucieux d'intéresser l'opinion à la science pour assurer les débouchés de leurs ouvrages de vulgarisation. Au siècle suivant les chercheurs s'investissent plus volontiers dans la communication scientifique : certains pour restaurer leur légitimité (celle des chimistes ayant souffert de la guerre des gaz ou celle des physiciens d'Hiroshima), d'autres pour « embrigader » l'opinion et obtenir qu'elle fasse pression en leur faveur sur les autorités. De la popularisation à la vulgarisation, puis à la communication, il faut noter l'évolution des termes mais ne

\footnotetext{
${ }^{1}$ Ces deux rapports peuvent être rattachés schématiquement au rationalisme des Lumières et au positivisme, ainsi qu'aux écoles d'épistémologie qu'ils ont inspirées (et dont les figures tutélaires sont Kuhn et Feyerabend pour la première, Bachelard et Popper pour la seconde).
} 
pas s'y tromper. Tous répondent à la même logique diffusionniste, selon laquelle il appartient aux producteurs du savoir de le propager vers des consommateurs nécessairement passifs, et qui culmine depuis une cinquantaine d'années avec la dénonciation régulière des maux de l'opinion et des progrès de l'irrationalisme (spécificité française semble-t-il).

Abordons maintenant la partie programmatique de l'ouvrage. Bernadette Bensaude-Vincent y précise d'abord quel risque la conviction qu'il existe un fossé infranchissable entre la science et l'opinion fait courir à la démocratie : s'en remettre absolument à l'autorité des experts prive les citoyens du droit d'exercer leur jugement et de décider de leur avenir. En guise de remède, elle propose de les évaluer symétriquement selon leurs caractéristiques respectives. Pour la première, cela consiste à rappeler que les connaissances scientifiques ne sont pas absolues mais socialement produites et situées, et qu'elles sont valables dans des circonstances expérimentales souvent éloignées de la réalité ordinaire - de quoi limiter la prétention des experts à régenter la vie quotidienne. Pour la seconde, à préciser qu'elle dispose de son propre système d'évaluation, orienté vers l'efficacité dans l'action (plutôt que vers la clôture des controverses, ce qui favorise le maintien de la pensée dans l'éveil) et enraciné dans les solides savoirs pratiques des acteurs. Entre elles paraît ainsi s'ouvrir un espace de complémentarité, dont l'auteur espère que la reconnaissance aboutisse à la signature d'un « accord de paix » instaurant des relations véritablement démocratiques entre la communauté scientifique, les pouvoirs publics et les citoyens. Dans la postface originale dont cette édition est suivie, elle reprend ce thème pour se féliciter de son actualité et des progrès réalisés depuis la première édition de 1999.

Que penser finalement de cet ouvrage ? Il forme un ensemble contrasté, et pour tout dire inégal. Ses deux premiers chapitres sont fort intéressants : bien documentés mais d'un abord facile (les nombreuses références sont placées en notes et n'alourdissent pas la lecture), ils s'attachent autant à définir précisément les concepts qu'à lever les ambiguïtés de leurs occurrences historiques, et permettent de jeter un regard neuf sur la formation de la science contemporaine. Seule la typographie parfois négligée vient en troubler la lecture. Le troisième chapitre et la postface en revanche, sont nettement moins convaincants. On y reconnaît sans peine les thèses popularisées depuis deux décennies par un courant de pensée issu de la sociologie des sciences, s'appuyant sur les concepts de controverse ou de démocratie technique. S'il n'est pas lieu de les discuter ici (le lecteur curieux en trouvera un aperçu en ligne dans un échange entre Yannick Barthe et Jean-Baptiste Fressoz ${ }^{2}$, et une analyse plus serrée dans un récent ouvrage collectif ${ }^{3}$ ), on regretta certaines lacunes méthodologiques qui dans ce livre en affaiblissent la démonstration. Citons parmi les plus embarrassantes le manque de rigueur dans l'usage des concepts (aux notions de science et d'opinion sont implicitement substituées celles d'expertise et d'opinion éclairée), l'indigence du matériau empirique (le propos s'appuie quasi-exclusivement sur la comparaison entre deux anecdotes rapportées sur les philosophes Démocrite et Thalès), et l'absence totale de réflexivité (comment valoriser " l'opinion droite » sans dé-

\footnotetext{
${ }^{2}$ Barthe Yannick, « Aux risques du passé » et Fressoz Jean-Baptiste, « L'apocalypse joyeuse : réfléchir sans rien changer », La Vie des idées, 19 octobre 2012.

URL : http://www.laviedesidees.fr/Aux-risques-du-passe.html

${ }^{3}$ Bourg Dominique, Joly Pierre-Benoît, Kaufmann Alain (dir.), Du risque à la menace. Penser la catastrophe, PUF, 2013, 282 p.
} 
noncer du même coup « les opinions tordues » et se heurter de nouveau à l'opposition initialement dénoncée ?). On ne peut que souhaiter que Bernadette BensaudeVincent prenne au sérieux son projet « doxologique » et s'attache à corriger ces lacunes. En attendant, on recommandera sans hésitation de consulter les deux premiers chapitres de L'opinion publique et la science, et sans remords de s'épargner le troisième. 